\title{
The role of the massless phantom term in the stability of a non-topological soliton solution
}

\author{
M. Mohammadi \\ Physics Department, Persian Gulf University, Bushehr 75169, Iran:*
}

\begin{abstract}
We intend to introduce classically a special Lagrangian density in such a way that, firstly, it leads to a special non-topological solitary wave solution, secondly, the stability of that is guaranteed properly, and thirdly, its dominant dynamical equations reduce to the standard nonlinear KleinGordon equations. For these purposes, we have to consider a new term in the Lagrangian density, whose role is like a massless phantom that surrounds the special solitary wave solution and resists any change in its internal structure.
\end{abstract}

\footnotetext{
* physmohammadi@pgu.ac.ir
} 
Keywords : soliton, solitary wave, Klein-Gorgon, nonlinear, Q-ball, stability, massless, phantom.

\section{INTRODUCTION}

In many of known physical models in the quantum field theory, dynamical field equations are introduced as standard (nonlinear) Klein-Gordon or (nonlinear) Klein-Gordon-like equations. Each of these equations with a set of particular constants just is used for a special type of fundamental particles with specific characteristics. For example, the components of Dirac equation (as some Klein-Gordon equations) were initially introduced to describe the quantum behavior of electrons and positrons. The Dirac equation, with different constants, is used for neutrinos and muons separately. Also, for the known Higgs particles, the dominant dynamical equation is a complex nonlinear Klein-Gordon (KG) equation. Nevertheless, the truth and the nature of the particle itself remain unknown in the standard quantum field theory. In other words, the quantum field theory is just a mathematical structure that provides a correct probabilistic relationship between initial conditions and the output results. Many of the properties of the fundamental particles in the quantum field theory (such as mass and charge) are based solely on the results that obtained in the laboratory and are used manually to obtain the appropriate dynamical equations in this standard and successful theory. In fact, the quantum field theory, despite many of its significant successes in predicting the probability behavior of particles, in many other respects, can not explain many of other questions. For example, why should the mass and charge of a fundamental particle, such as an electron, be specific numbers? Or, why is there just a single Planck constant $\hbar$ for the all particles of the nature? These are some of the questions that have not been yet explained properly.

Classically, for any arbitrary system of the fields, there are some PDEs which are called the dynamical wave equations. In general, theses PDEs have infinite solutions but a special solution with minimum rest energy is an important one, the so-called a soliton solution [1 5]. A soliton solution in many respects is in accordance with our classical sense of the particle concept, i.e. a prominent stable profile of the field in the space whose behavior in the collisions is fully in line with well-known standard theories. For example, for the real nonlinear KG systems with kink (antikink) solutions, many of the expected properties, that 
we expect to satisfy for any real classical relativistic particle, are satisfied properly [1, 5 -27]. In fact, the theory of the classical fields with soliton solutions is a hopeful point for some researchers to answer the unanswered questions that remain in the quantum field theory. The stability condition for soliton solutions has forced many researchers to look for models that result topological solutions [1, 5, 28-33]. Basically, the topological property causes the stability of the soliton solution to be automatically guaranteed. Instead, among the models with non-topological solutions [27, 34 51], no model has been yet introduced that can lead to a soliton solution with minimum energy. The importance of the non-topological solutions is that it is easy to imagine a multi-particle solution just by adding them when they are far enough from each other. In the case of topological solutions to have a multi-particle solution, the situation is usually very complicated and sometimes impossible. Note that, the new model in this paper is introduced in $1+1$ dimension just for simplicity, but it can be extended to $3+1$ dimensions in a similar way.

In this paper, inspire by what we have learned in the quantum field theory, we try to show how it is possible to introduce a classical model of the relativistic fields in such a way that it leads to a special non-topological soliton solution with a special type of the standard nonlinear KG equations as its dominant dynamical equations. We show that in order to achieve these demands, we have to add a new term to a standard nonlinear KG Lagrangian density which behaves like a massless phantom that surrounds its special solitary wave solution and obstructs any arbitrary change in the internal structure of that. In other words, we want it acts like a stability catalyzer and its role be hidden when the special solitary wave solution is free and non-deformed. This new special classical relativistic field model is an example of the extended nonlinear KG field systems that are introduced in this paper generally.

The organization of this paper is as follows: In Section III, we introduce the standard and the extended (nonlinear) KG systems for scalar fields. In Section III, in general, we will introduce an extended nonlinear KG system with a special non-topological soliton solution. In Section IV, the stability of the special soliton solution is considered under the small variations. The last section is devoted to summary and conclusions. 


\section{EXTENDED NONLINEAR KLEIN-GORDON SYSTEMS}

In the standard relativistic (classical and quantum) field theory, the standard forms of the Lagrangian densities for the scalar fields are expressed as follows:

$$
\mathcal{L}=\sum_{j \geqslant i}^{N} \sum_{i=1}^{N} \alpha_{i j}\left(\phi_{1}, \cdots, \phi_{N}\right) S_{i j}-V\left(\phi_{1}, \cdots, \phi_{N}\right),
$$

here $\phi_{i}$ 's are $N$ independent scalar fields, coefficients $\alpha_{i j}$ 's and potential term $V$ all are functions of the fields, scalars $S_{i j}=S_{j i}=\partial_{\mu} \phi_{i} \partial^{\mu} \phi_{j}$ are named kinetic scalar terms. In fact, such kind of Lagrangian densities represent the standard (nonlinear) KG systems. In other words, the formulas of the Lagrangian density of the standard (nonlinear) KG systems are linearly expanded in terms of the kinetic scalar terms $S_{i j}$. Note that, the various linear combinations of $S_{i j}$ 's, in accordance with the standard (11), are ones that result a real Lagrangian density. However, according to the standard (1), depending on the arbitrary choices of the coefficients $\alpha_{i j}$ 's and potential term $V$, it can be possible to introduce infinite (nonlinear) KG system. For example, if we deal with a complex scalar field $\phi$, then $\phi_{1}=\phi, \phi_{2}=\phi^{*}$ and the allowed kinetic scalar terms are $S_{11}=\partial_{\mu} \phi \partial^{\mu} \phi, S_{22}=\partial_{\mu} \phi^{*} \partial^{\mu} \phi^{*}$ and $S_{12}=\partial_{\mu} \phi \partial^{\mu} \phi^{*}$. Accordingly, for a complex scalar field $\phi$, the well known systems with the non-topological solitary wave solutions (Q-balls) were introduced as follows [27, 34- 51]:

$$
\mathcal{L}=\partial_{\mu} \phi^{*} \partial^{\mu} \phi-V(R)
$$

where $\alpha_{11}=\alpha_{22}=0, \alpha_{12}=1$ and $R=|\phi|=\sqrt{\phi \phi^{*}}$. Q-balls, unlike kinks and anti-kinks, are non-topological solitary wave solutions which are not energetically stable. In general, it was shown that Q-balls have the minimum rest energy among the other solutions with the same electrical charge, but it is not a sufficient condition for the stability [38, 40].

In an equivalent representation, instead of a complex scalar field $\phi$, using the polar representation of the fields

$$
\phi(x, t)=R(x, t) \exp [i \theta(x, t)]
$$

leads to a new form of the systems with the Q-ball solutions, i.e.

$$
\mathcal{L}=\left(\partial^{\mu} R \partial_{\mu} R\right)+R^{2}\left(\partial^{\mu} \theta \partial_{\mu} \theta\right)-V(R)
$$

where $\phi_{1}=R, \phi_{2}=\theta, S_{11}=\partial_{\mu} R \partial^{\mu} R, S_{22}=\partial_{\mu} \theta \partial^{\mu} \theta, S_{12}=\partial_{\mu} R \partial^{\mu} \theta, \alpha_{11}=1, \alpha_{22}=R^{2}$ and $\alpha_{12}=0$. Therefore, a special relativistic field system can be introduced via many different, 
but equivalent, representation. For each of these equivalent representation, the coefficients $\alpha_{i j}$ 's and the kinetic scalar terms $S_{i j}$ 's would be different.

If the relativistic Lagrangian densities of the scalar fields are not linear combination of $S_{i j}$ 's, we can call them extended nonlinear $K G$ systems. Namely, for a real scalar field $\phi_{1}=\varphi$ with a single kinetic scalar term $S_{11}=S=\partial_{\mu} \varphi \partial^{\mu} \varphi$, the following Lagrangian densities are two examples of the extended nonlinear KG systems:

$$
\begin{aligned}
& \mathcal{L}=\varphi S+S^{2}-V(\varphi), \\
& \mathcal{L}=[S-V(\varphi)]^{2}
\end{aligned}
$$

Moreover, for a complex field $\phi$ in the polar representation, i.e. $\phi_{1}=R$ and $\phi_{2}=\theta$, the following Lagrangian densities are again two examples of the extended nonlinear KG systems:

$$
\begin{aligned}
& \mathcal{L}=\left(\partial^{\mu} R \partial_{\mu} R\right)+R^{2}\left(\partial^{\mu} \theta \partial_{\mu} \theta\right)^{3}=\left(\mathcal{S}_{12}\right)+R^{2}\left(\mathcal{S}_{22}\right)^{3} \\
& \left.\mathcal{L}=\left(\partial^{\mu} R \partial_{\mu} R\right)\left(\partial^{\mu} \theta \partial_{\mu} \theta\right)-V(R)=\left(\mathcal{S}_{11}\right)\left(\mathcal{S}_{22}\right)-V(R)\right) .
\end{aligned}
$$

The extended nonlinear KG systems, due to their nonlinear dependence on the kinetic

scalars $S_{i j}$, undoubtedly end up with nonlinear dynamical equations of motion which are too complicated and are unlikely to be used until now in well-known physical models. Note that, the pervious Eqs. (5)-(8) are just some arbitrary examples of the extended nonlinear KG systems and there is not any other meaning, i.e. they should not be considered as some extended field systems with some special solutions or some thing else. Of course, in general, such Lagrangian densities can be called nonstandard Lagrangian (NSL) densities too [52 56]. There are many works which were dealing with such systems among which one can mention the works of Riazi and his colleagues [32, 33] and El-Nabulsi [55, 56]. However, in the next section we will introduce a special extended nonlinear KG system which leads to a single non-topological soliton solution.

\section{INTRODUCING AN EXTENDED NONLINEAR KLIEN-GORDON SYSTEM WITH A SPECIAL NON-TOPOLOGICAL SOLITON SOLUTION}

Here, at first step, we are going to introduce a standard complex non-linear KG system with a special Q-ball solution. For example, if we chose a special potential

$$
V(R)=-R^{6}+R^{4}+100 R^{2}
$$


for Lagrangian density (4), then the related equations of motion would be

$$
\begin{aligned}
& \square R-R\left(\partial^{\mu} \theta \partial_{\mu} \theta\right)=-\frac{1}{2} \frac{d V}{d R}, \\
& \partial_{\mu}\left(R^{2} \partial^{\mu} \theta\right)=2 R\left(\partial_{\mu} R \partial^{\mu} \theta\right)+R^{2}\left(\partial^{\mu} \partial_{\mu} \theta\right)=0 .
\end{aligned}
$$

It is easy to show that these dynamical equations have a special Q-ball solution as follows:

$$
\phi=R_{s}(x) e^{i \omega_{s} t}=\frac{1}{\sqrt{1+x^{2}}} e^{i 10 t}
$$

where $\omega_{s}=10$ can be called the rest frequency of this special solution. This solution is at rest, to obtain the moving version of that, if it moves at velocity of $v$, it can be easily accomplished using a relativistic boost:

$$
\phi=\frac{1}{\sqrt{1+\gamma^{2}(x-v t)^{2}}} e^{i k_{\mu} x^{\mu}},
$$

where $\gamma=\left(1-v^{2}\right)^{-1 / 2}$ and $k^{\mu} \equiv(\omega, k)$, provided $\omega=\omega_{s} \gamma$ and $k=\omega v$. Note that, in this paper, for simplicity, we take the speed of light equal to one $(c=1)$.

The energy density function belonging to Lagrangian density (41) can be easily obtained:

$$
\varepsilon(x, t)=\left[\dot{R}^{2}+R^{\prime 2}+R^{2}\left(\dot{\theta}^{2}+\theta^{\prime 2}\right)+V(R)\right],
$$

where prime and dot are used to specify the space and time derivatives respectively. Since for the non-moving special Q-ball solution (12) $\dot{R}_{s}=\theta_{s}^{\prime}=0$, therefore the rest energy of that would be

$$
E_{o}=\int_{-\infty}^{+\infty}\left[R_{s}^{\prime 2}+\omega_{s}^{2} R_{s}^{2}+V\left(R_{s}\right)\right] d x .
$$

It is easy to show that the non-topological Q-ball solution (12) is not stable under any arbitrary small deformation. For example, if we fix the phase function $\theta=\omega_{s} t$, according to Eqs. (15) and (9), it is easy to show any small variation in $R_{s}$ with $\delta R_{s}<0$, yields a small reduction in the related total energy. In other words, the Q-ball solutions, such as (12), are not energetically stable objects at all.

Our main goal in this paper is that to find a proper additional term $(F)$ for the original Lagrangian density (44) in such a way that, like a stability catalyzer, guarantees the energetically stability of the special Q-ball solution (12) and dose not have any role in the dominant dynamical equations for this special solution, i.e. the dynamical equations just for the special Q-ball solution (12) remain the same standard original equations (10) and 
(11). In other words, we want to introduce a new extended nonlinear Lagrangian density as follows

$$
\mathcal{L}_{N}=\mathcal{L}+F=\left[\partial^{\mu} R \partial_{\mu} R+R^{2}\left(\partial^{\mu} \theta \partial_{\mu} \theta\right)-V(R)\right]+F,
$$

in such a way that the pervious solitary wave function (12) being a solution again. Moreover, we expect the new dynamical equations of this new extended system (16) reduce to the same original ones (10 and 11) just for the special solitary wave solution (12). In general, since the Lagrangian density (16) must be scalar, so the new unknown term $F$ can be only a function of the allowed scalars. Allowed scalars are the module field $R$, the phase field $\theta$, and the kinetic scalar terms $\partial_{\mu} R \partial^{\mu} R, \partial_{\mu} \theta \partial^{\mu} \theta$ and $\partial_{\mu} R \partial^{\mu} \theta$. To keep the charge conservation law again, $F$ should not depend explicitly on $\theta$. However, the new form of the dynamical equations of the new Lagrangian density (16) are obtained as follows:

$$
\begin{aligned}
& \square R-R\left(\partial^{\mu} \theta \partial_{\mu} \theta\right)+\frac{1}{2} \frac{d V}{d R}+\frac{1}{2}\left[\frac{\partial}{\partial x^{\mu}}\left(\frac{\partial F}{\partial\left(\partial_{\mu} R\right)}\right)-\left(\frac{\partial F}{\partial R}\right)\right]=0 \\
& \partial_{\mu}\left(R^{2} \partial^{\mu} \theta\right)+\frac{1}{2}\left[\frac{\partial}{\partial x^{\mu}}\left(\frac{\partial F}{\partial\left(\partial_{\mu} \theta\right)}\right)\right]=0 .
\end{aligned}
$$

Based on the well-known relations in the standard classical field theory, it is easy to find the energy density function of the system (16):

$$
\varepsilon(x, t)=\varepsilon_{o}+\varepsilon_{F}=\left[\dot{R}^{2}+R^{\prime 2}+R^{2}\left(\dot{\theta}^{2}+\theta^{\prime 2}\right)+V(R)\right]+\left[\dot{R} \frac{\partial F}{\partial \dot{R}}+\dot{\theta} \frac{\partial F}{\partial \dot{\theta}}\right]
$$

Where $\varepsilon_{o}$ corresponds to the original Lagrangian density (4) and $\varepsilon_{F}$ is related to the unknown new added term $F$. As we have mentioned, for the special solution (12), we expect the equations of motion (17) and (18) reduce to the same original standard nonlinear KG equations (10) and (11) respectively. In other words, for the new system (16), we expect the localized wave function (12) being a solution again, and for that the terms which are expressed in $F$ and its derivatives, all be equal to zero. This means that the dominant dynamical equations over the special solution (12) are the same standard equations (10) and (11). Therefore, we expect $F$ and all its derivatives that appear in the above equations would be zero simultaneously for the special solution (12). This goal is only possible if $F$ is considered as a function of the powers of three special scalars; the scalars that all are zero for the special solution (12). It is easy to show that these scalars are:

$$
\begin{aligned}
& \mathcal{S}_{1}=\partial_{\mu} \theta \partial^{\mu} \theta-\omega_{s}^{2}, \\
& \mathcal{S}_{2}=\partial_{\mu} R \partial^{\mu} R-R^{6}+R^{4}, \\
& \mathcal{S}_{3}=\partial_{\mu} R \partial^{\mu} \theta .
\end{aligned}
$$


For example, if one considers $F=F\left(\mathcal{S}_{1}^{n}, \mathcal{S}_{2}^{n}, \mathcal{S}_{3}^{n}\right)$, it leads to

$$
\begin{aligned}
& \frac{\partial}{\partial x^{\mu}}\left(\frac{\partial F}{\partial\left(\partial_{\mu} R\right)}\right)=\sum_{i=1}^{3}\left[n(n-1) \mathcal{S}_{i}^{(n-2)} \frac{\partial \mathcal{S}_{i}}{\partial x^{\mu}} \frac{\partial \mathcal{S}_{i}}{\partial\left(\partial_{\mu} R\right)} \frac{\partial F}{\partial Z_{i}}+n \mathcal{S}_{i}^{(n-1)} \frac{\partial}{\partial x^{\mu}}\left(\frac{\partial \mathcal{S}_{i}}{\partial\left(\partial_{\mu} R\right)} \frac{\partial F}{\partial Z_{i}}\right)\right] \\
& \frac{\partial F}{\partial R}=\sum_{i=1}^{3}\left[n \mathcal{S}_{i}^{(n-1)} \frac{\partial \mathcal{S}_{i}}{\partial R} \frac{\partial F}{\partial Z_{i}}\right] \\
& \frac{\partial}{\partial x^{\mu}}\left(\frac{\partial F}{\partial\left(\partial_{\mu} \theta\right)}\right)=\sum_{i=1}^{3}\left[n(n-1) \mathcal{S}_{i}^{(n-2)} \frac{\partial \mathcal{S}_{i}}{\partial x^{\mu}} \frac{\partial \mathcal{S}_{i}}{\partial\left(\partial_{\mu} \theta\right)} \frac{\partial F}{\partial Z_{i}}+n \mathcal{S}_{i}^{(n-1)} \frac{\partial}{\partial x^{\mu}}\left(\frac{\partial \mathcal{S}_{i}}{\partial\left(\partial_{\mu} \theta\right)} \frac{\partial F}{\partial Z_{i}}\right)\right]
\end{aligned}
$$

where $Z_{i}=\mathcal{S}_{i}^{n}$. It is easy to understand that if $n \geq 3$, then all of these terms would be zero for the special solution (12). In general, it can be shown that the all combinations according to the following series have the desired wanted features:

$$
F=\sum_{n_{3}=0}^{\infty} \sum_{n_{2}=0}^{\infty} \sum_{n_{1}=0}^{\infty} a\left(n_{1}, n_{2}, n_{3}\right) \mathcal{S}_{1}^{n_{1}} \mathcal{S}_{2}^{n_{2}} \mathcal{S}_{3}^{n_{3}},
$$

provided $\left(n_{1}+n_{2}+n_{3}\right) \geq 3$. Here, coefficients $a\left(n_{1}, n_{2}, n_{3}\right)$ are some arbitrary functions of the allowed scalars (except $\theta$ ). Accordingly, the new purposed system (16) is essentially an extended nonlinear Klien-Gordon system. In fact, it consists of two parts, the original part $\mathcal{L}$ which is a standard KG system, and the additional term $F$, which must be functions of the powers of the kinetic scalars.

For some special choices of the series (23), the stability of the special solution (12) is guaranteed properly. For example, first let us consider three linear independent combinations of scalars $\mathcal{S}_{1}, \mathcal{S}_{2}$ and $\mathcal{S}_{3}$ as follows:

$$
\begin{aligned}
& \mathcal{K}_{1}=R^{2} \mathcal{S}_{1}, \\
& \mathcal{K}_{2}=R^{2} \mathcal{S}_{1}+\mathcal{S}_{2}, \\
& \mathcal{K}_{3}=R^{2} \mathcal{S}_{1}+\mathcal{S}_{2}+2 R \mathcal{S}_{3} .
\end{aligned}
$$

Then, the proper additional functional $F$ can be introduced as follow:

$$
F=\sum_{i=1}^{3} A_{i}\left(\mathcal{K}_{i}\right)^{3}
$$

In which $A_{i}$ 's $(i=1,2,3)$ are only three large constants. However, for this particular choice (27), the corresponding energy density function is

$$
\begin{aligned}
\varepsilon(x, t)= & {\left[\dot{R}^{2}+R^{\prime 2}+R^{2}\left(\dot{\theta}^{2}+\theta^{\prime 2}\right)+V(R)\right]+} \\
& \sum_{i=1}^{3}\left[3 A_{i} C_{i} \mathcal{K}_{i}^{2}-A_{i} \mathcal{K}_{i}^{3}\right]=\varepsilon_{o}+\varepsilon_{1}+\varepsilon_{2}+\varepsilon_{3},
\end{aligned}
$$


which is divided into four separate parts. Coefficients $C_{i}$ 's are

$$
C_{i}=\frac{\partial \mathcal{K}_{i}}{\partial \dot{\theta}} \dot{\theta}+\frac{\partial \mathcal{K}_{i}}{\partial \dot{R}} \dot{R}=\left\{\begin{array}{cc}
2 R^{2} \dot{\theta}^{2} & \mathrm{i}=1 \\
2\left(\dot{R}^{2}+R^{2} \dot{\theta}^{2}\right) & \mathrm{i}=2 \\
2(\dot{R}+R \dot{\theta})^{2} & \mathrm{i}=3 .
\end{array}\right.
$$

After a straightforward calculation, given that $\omega_{s}^{2}=100$, one can obtain:

$$
\begin{aligned}
& \varepsilon_{1}=\mathcal{K}_{1}^{2} A_{1}\left[5 R^{2} \dot{\theta}^{2}+R^{2} \theta^{\prime 2}+100 R^{2}\right] \geq 0, \\
& \varepsilon_{2}=\mathcal{K}_{2}^{2} A_{2}\left[5 R^{2} \dot{\theta}^{2}+5 \dot{R}^{2}+R^{2} \theta^{\prime 2}+U(R)\right] \geq 0, \\
& \varepsilon_{3}=\mathcal{K}_{3}^{2} A_{3}\left[5(R \dot{\theta}+\dot{R})^{2}+\left(R \theta^{\prime}+R^{\prime}\right)^{2}+U(R)\right] \geq 0,
\end{aligned}
$$

in which

$$
U(R)=R^{6}-R^{4}+100 R^{2} .
$$

This function (33) is always ascending and bounded from below by zero. Therefore, all terms in $\varepsilon_{1}, \varepsilon_{2}$ and $\varepsilon_{3}$ are positive definite. For the special solution (12) and vacuum state $(R=0)$, all $\varepsilon_{i}$ 's $(i=1,2,3)$ would be zero simultaneously. It is reminded that $\mathcal{K}_{i}$ 's, like the scalars $\mathcal{S}_{i}$ 's, for the special solution (12), all would be zero simultaneously. Since, for the special solution (12) the energy contribution belongs to the additional term $F$ is practically zero (i.e. $\mathcal{K}_{i}=0$ then $\varepsilon_{F}=\sum_{i=1}^{3} \varepsilon_{i}=0$ ), that is why we call the additional term $F$ "phantom term".

In general, since there are two independent scalar fields $R$ and $\theta$, and depending on them three independent scalars $\mathcal{K}_{1}, \mathcal{K}_{2}$ and $\mathcal{K}_{3}$ are considered, therefore, except for the non-trivial solitary wave solution (12), it is not possible to find any other space-time functions $R(x, t)$ and $\theta(x, t)$ for which $\mathcal{K}_{i}$ 's all being zero simultaneously. Hence, for the other solutions of the new extended system (16 with 27), it is never possible for three independent scalars $\mathcal{S}_{i}$ 's or $\mathcal{K}_{i}$ 's to be zero simultaneously. In other words, for the other solutions of the new system (16 with 27), always at least one of the scalars $\mathcal{K}_{i}$ 's is a nonzero function, and then if constants $A_{i}$ 's are considered to be large numbers, at least one of the functions $\varepsilon_{i}$ 's $(i=1,2,3)$ becomes a non-zero large function, which implies that the energy of the other solutions would be always larger than the rest energy of the special solution (12). Accordingly, the definition of the new extended system (27) for large values of $A_{i}$ 's, causes the special solution (12) turns to a soliton solution with the minimum rest energy among the other solutions 
of the new extended system (27). Note that, the first term of the energy density function $\left(\varepsilon_{o}\right)$ has not considered yet in the stability consideration. In the next section, we show that the contribution of this term (i.e. $\varepsilon_{o}$ ) is so small, compared to the other terms $\varepsilon_{i}$ 's, that essentially can be ignored in the stability consideration, provided constants $A_{i}$ 's are considered to be large numbers.

\section{STABILITY UNDER SMALL VARIATIONS}

In this section, we want to study the stability of the special solution (12) for the small deformations. In general, a little deformed non-moving solitary wave solution can be presented as follows:

$$
R(x, t)=R_{s}(x)+\delta R(x, t), \quad \theta(x, t)=\theta_{s}(t)+\delta \theta=\omega_{s} t+\delta \theta(x, t)
$$

where $R_{s}(x)=1 / \sqrt{1+x^{2}}$ and $\theta_{s}=\omega_{s} t$, and $\delta R$ and $\delta \theta$ are any permissible small spacetime functions. Now, by inserting this deformed solution (34) in the first part of the energy density $\varepsilon_{o}(28)$ and keeping the terms in order of $\delta R$ and $\delta \theta$, then it yields:

$$
\begin{aligned}
& \varepsilon_{o}(x, t)=\varepsilon_{o s}(x)+\delta \varepsilon_{o}(x, t) \approx\left[R_{s}^{\prime 2}+R_{s}^{2} \omega_{s}^{2}+V\left(R_{s}\right)\right]+ \\
& 2\left[R_{s}^{\prime}\left(\delta R^{\prime}\right)+R_{s}(\delta R) \omega_{s}^{2}+R_{s}^{2} \omega_{s}(\delta \dot{\theta})+\frac{1}{2} \frac{d V\left(R_{s}\right)}{d R_{s}}(\delta R)\right] .
\end{aligned}
$$

Note that for the special non-moving solution (12), $\dot{R}_{s}=0, \theta_{s}^{\prime}=0$ and $\dot{\theta}_{s}=\omega_{s}$. It is clear that $\delta \varepsilon_{o}$ is not necessarily an absolute positive function. Hence, it may take negative values at some space-time points for some permissible variations $\delta R$ and $\delta \theta$. Now, we do this for the other energy density parts (28):

$$
\begin{aligned}
& \varepsilon_{i}(x, t)=\varepsilon_{i s}+\delta \varepsilon_{i}=\delta \varepsilon_{i}=\left[3 A_{i}\left(C_{i s}+\delta C_{i}\right)\left(\mathcal{K}_{i s}+\delta \mathcal{K}_{i}\right)^{2}-A_{i}\left(\mathcal{K}_{i s}+\delta \mathcal{K}_{i}\right)^{3}\right]= \\
& {\left[3 A_{i}\left(C_{i s}+\delta C_{i}\right)\left(\delta \mathcal{K}_{i}\right)^{2}-A_{i}\left(\delta \mathcal{K}_{i}\right)^{3}\right] \approx\left[3 A_{i} C_{i s}\left(\delta \mathcal{K}_{i}\right)^{2}-A_{i}\left(\delta \mathcal{K}_{i}\right)^{3}\right] \approx\left[6 A_{i} \omega_{s}^{2}\left(\delta \mathcal{K}_{i}\right)^{2}\right] \geqslant 0}
\end{aligned}
$$

Note that, for the special solitary wave solution (12): $\varepsilon_{i s}=0, \mathcal{K}_{i s}=0$ and $C_{i s}=2 \omega_{s}^{2}$. As we expected, according to the previous equation (36) $), \delta \varepsilon_{i}$ 's $(i=1,2,3)$ all are always positive definite.

The variation of the total energy density function $(\delta \varepsilon)$ would be equal to the sum of the changes in the all four separate parts, namely $\delta \varepsilon=\delta \varepsilon_{o}+\sum_{i=1}^{3} \delta \varepsilon_{i}$. Since $\delta \varepsilon_{o}$ is not 
necessarily positive and $\delta \varepsilon_{i}$ 's are always positive, therefore, the difference between the order of magnitudes of $\sum_{i=1}^{3} \delta \varepsilon_{i}$ and $\delta \varepsilon_{o}$ is important for various small deformations (34). In general, it can be easily shown that $\delta \mathcal{K}_{i}$ 's and $\delta C_{i}$ 's are in the order of the first power of the variations $\delta R$ and $\delta \theta$. Therefore, according to the result (36), $\delta \varepsilon_{i}$ 's are in the order of $A_{i}(\delta R)^{2}, A_{i}(\delta \theta)^{2}$ and $A_{i}(\delta R \delta \theta)$, while $\delta \varepsilon_{o}$, as mentioned above (35), is only in the order of $\delta R$ and $\delta \theta$.

On this basis, if constants $A_{i}$ 's are considered to be large numbers, it can be easily understood that the special solution (12) effectively leads to a stable solution under the influence of the small variations. In fact, there is approximately an small certain amount for the order of the magnitude of the small variations $\delta R$ and $\delta \theta$ in such a way that if the maximum of them be larger than this certain amount, $\delta \varepsilon$ would be positive and then the special solution is called a stable object for such variations. For example, if we assume a system with $A_{i}=10^{40}$, then only for the variations that $\max \{O(\delta R), O(\delta \theta)\}<10^{-20}$, the special solution (12) dose not have the condition of the stability. In other words, for these very small variations (i.e. $\max \{O(\delta R), O(\delta \theta)\}<10^{-20}$ ), the total energy density variation $(\delta \varepsilon)$ may take negative values. But note that, these variations are so small which physically can be ignored in the stability considerations. However, if the order of the magnitude of one of the variations $\delta R$ and $\delta \theta$ be larger than $10^{-20}$ (i.e. $\max \{O(\delta R), O(\delta \theta)\}>10^{-20}$ ), then $\delta \varepsilon$ would be positive and the stability is guaranteed appreciably for such small variations.

Since the special solution (12) does not have the stability condition for very small variations, it is a sign of the fact that the dominant dynamical equation for the special solution (12) is the same standard nonlinear KG equations (10) and (11). In other words, for very small unimportant variations, the condition of stability is violated (i.e. $\left|\delta \epsilon_{o}\right| \geq \sum_{i=1}^{3} \delta \varepsilon_{i}$ ), and the role of terms which containing functional $F$, in comparison with other terms, would be ineffective.

Through a biologic example, we try to make the story better. Understanding the unstable nature of the special solution (12) for very low variations, less than the certain amount, is like a chicken in the egg. Chicken inside the egg can slightly move its head, hands and feet, and it does not necessarily have a permanent fixed status. But, allowed movements for chicken within egg, due to egg shell, are subject to severe constraints, for example, it can not send out their feet through the egg shell. In other words, regardless some unimportant movements inside the egg, due to the solid egg shell, the chicken has to be in the same shape 
of the egg. For this simulation, firstly, the phantom term $F$ has the role of the egg shell, of course a massless and very solid shell, i.e. the phantom term $F$ requires that the particle shape (12) always stays in a specified format; secondly, the chicken itself is similar to the same special solution (12); and thirdly, the internal permissible movements of the chicken inside the egg are like some permissible so small variations of the special solution (12) for which $\delta \varepsilon<0$. Briefly, the new phantom term (27) in the new extended system (16) behaves like a stability catalyzer and does not have any role in the other observable such as rest mass and charge.

Some one may think that this model is an artificial model which is properly tuned to satisfied all wanted features specially for a purposed solitary wave solution (12). But note that, firstly; many of the models in the standard quantum field theory are artificial, i.e. many people try to tune them manually so that lead to desired results and expectations, secondly; it have not been yet a classical relativistic field model which leads to an energetically stable non-topological soliton solution and this one is the first, thirdly; the required features which we introduced in the beginning of the section [II for the special particle-like function (12), forced us to reach this strange model, i.e. it is unique and we could not find another completely different one which satisfied the all wanted features properly.

\section{CONCLUSION}

Considering the above points, it is theoretically possible to speak of a special nontopological soliton solution (12) for which the dominant dynamical equation would be a special kind of the standard nonlinear KG equations. This was done if one add a new proper scalar term $(F)$ to the original KG Lagrangian density (4). This new term behaves like a massless phantom which surrounds the particle-like solution (12) and resists any significant changes in its internal structure. For a free solitary wave solution (12) or one whose internal structure is deformed very small, the dominant dynamical equations are the same standard nonlinear KG equations (10) and (11), and the contribution of the phantom term $F$ in its dominant dynamical equations is zero or negligible. In other words, just for this special solution (12), the complicated dynamical equations (17) and (18) reduce to the same standard nonlinear KG equations (10) and (11) respectively. But for some significant changes that are larger than a certain amount, the actual role of the added term $F$ is significant and 
resists such changes. In other words, the presence of such changes in the internal structure of the particle need high external energies. The power and ability of this new term $F$ for the stability of the special solution (12) is related to three constants $A_{i}(i=1,2,3)$ so that the larger values of those make the phantom term stronger. The role of the new (phantom) term $F$ for the special solution (12) just makes it stable, but does not affect its other physical properties, such as mass, charge, shape and the specific dominant dynamical equations, i.e. it acts like a stability catalyzer.

In general, the new system is one of the extended nonlinear KG systems that leads to very complicated dynamical equations. In general, such systems are thought to be nonstandard and distant in the standard theory of classical and quantum fields, but for the case presented in this paper, i.e. Eqs. (16) and (27), its solutions have energies larger than the rest energy of the special solitary wave solution (12). In other words, the special solitary wave solution (12) is truly a non-topological energetically stable soliton solution.

[1] R. Rajaraman, Solitons and Instantons (North Holland, Elsevier, Amsterdam, 1982).

[2] A. Das, Integrable Models (World Scientific, 1989).

[3] G. L. Lamb, Jr., Elements of Soliton Theory (John Wiley and Sons, USA, 1980).

[4] P. G. Drazin and R. S. Johnson, Solitons: an Introduction (Cambridge University Press, 1989).

[5] N. Manton, P. sutcliffe, Topological Solitons, (Cambridge University Press, 2004).

[6] D. K. Campbell and M. Peyrard, Kink-antikink interactions in the double sine-Gordon equation, Physica D, 19, 165 (1986).

[7] D. K. Campbell and M. Peyrard, Solitary wave collisions revisited, Physica D, 18, 47 (1986).

[8] D. K. Campbell, J. S. Schonfeld, and C. A. Wingate, Resonance structure in kink-antikink interactions in $\phi^{4}$ theory, Physica D, 9, 1 (1983).

[9] M. Peyrard and D. K. Campbell, Kink antikink interactions in a modified sine-Gordon model, Physica D, 9, 33 (1983).

[10] R. H. Goodman and R. Haberman, Kink-Antikink Collisions in the $\phi^{4}$ Equation: The ${ }^{n-}$ Bounce Resonance and the Separatrix Map, Siam J. Appl. Dyn. Syst, 4, 1195 (2005).

[11] O. V. Charkina, M. M. Bogdan, Internal Modes of Solitons and Near-Integrable HighlyDispersive Nonlinear Systems, Symmetry. Integr. Geom, 2, 047 (2006). 
[12] A. R. Gharaati, N. Riazi and F. Mohebbi, Internal modes of relativistic solitons, International Journal of Theoretical Physics, Int. J. Theor. Phys, 45, 53 (2006).

[13] M. Mohammadi and N. Riazi, Approaching integrality in bi-dimensional nonlinear field equations, Prog. Theor. Phys, 126, 237 (2011).

[14] M. Mohammadi and N. Riazi, The affective factors on the uncertainty in the collisions of the soliton solutions of the double field sine-Gordon system, Commun Nonlinear Sci Numer Simulat, 72, 176-193 (2019).

[15] V. A. Gani and A. E. Kudryavtsev, Kink-antikink interactions in the double sine-Gordon equation and the problem of resonance frequencies, Phys. Rev. E, 60, 3305 (1999).

[16] C. A. Popov, Perturbation theory for the double sine-Gordon equation, Wave Motion 42, 309 (2005).

[17] M. Peyravi, A. Montakhab, N. Riazi, and A. Gharaati, Interaction properties of the periodic and step-like solutions of the double-Sine-Gordon equation, Eur. Phys. J. B, 72, 269 (2009).

[18] M. Mohammadi, N. Riazi, and A. Azizi, Radiative Properties of Kinks in the $\sin ^{4}(\phi)$ System, Prog. Theor. Phys. 128, 615 (2012).

[19] P. Dorey, K. Mersh, T. Romanczukiewicz, and Y. Shnir, Kink-antikink collisions in the $\varphi^{6}$ model, Phys. Rev. Lett. 107, 091602 (2011).

[20] V. A. Gani, A. E. Kudryavtsev, and M. A. Lizunova, Kink interactions in the $(1+1)$ dimensional $\varphi^{6}$ model, Phys. Rev. D, 89, 125009 (2014).

[21] A. Khare, I. C. Christov, and A. Saxena, Successive phase transitions and kink solutions in $\phi^{8}, \phi^{10}$ and $\phi^{12}$ field theories, Phys. Rev. E, 90, 023208 (2014).

[22] A. Moradi Marjaneh, V. A. Gani, D. Saadatmand, Multi-kink collisions in the $\phi^{6}$ model, Journal of High Energy Physics, 2017, 28 (2017).

[23] D. Bazeia, E. Belendryasova, and V.A. Gani, Scattering of kinks of the sinh-deformed $\varphi^{4}$ model, Eur. Phys. J. C, 78, 340 (2018).

[24] V.A. Gani, A. Moradi Marjane, A. Askari, et al, Scattering of the double sine-Gordon kinks, Eur. Phys. J. C, 78, 345 (2018).

[25] P. Dorey, T. Romaczukiewicz, Resonant kinkantikink scattering through quasinormal modes, Physics Letters B, 779, 117-123 (2018).

[26] V. A. Gani, V. Lensky, M. A. Lizunova, Kink excitation spectra in the $(1+1)$-dimensional $\varphi^{8}$ model, JHEP, 147 (2015). 
[27] A. M. Wazwaz, Compactons, solitons and periodic solutions for some forms of nonlinear KleinGordon equations, Chaos, Solitons and Fractals, 28, 1005 (2006).

[28] T.H.R. Skyrme, A non-linear field theory, Proc. Roy. Soc, 260, 127, (1961).

[29] T. H. R. Skyrme, A unified field theory of mesons and baryons, Nucl. Phys, 31, 556 (1962).

[30] G. 't Hooft, Magnetic monopoles in unified gauge theories, Nuclear Physics, B79, 276 (1974).

[31] A. M. Polyakov, Particle spectrum in quantum field theory, JETP Lett. 20, 430 (1974).

[32] A. Vasheghani and N. Riazi, Isovector solitons and Maxwell's equations, Int. J. Theor. Phys, 35, 587 (1996).

[33] M. H. Mahzoon1 and N. Riazi, Nonlinear Electrodynamics and NED-Inspired Chiral Solitons, Int. J. Theor. Phys, 46, 823 (2007).

[34] A. M. Wazwaz, The tanh method for traveling wave solutions of nonlinear equations, Appl. Math. Comput, 154, 713 (2004).

[35] A. G. Panin, and M. N. Smolyakov, Problem with classical stability of U(1) gauged Q-balls, Phys. Rev. D, 95, 065006 (2017).

[36] A. Kovtun, E. Nugaev, and A. Shkerin, Vibrational modes of Q-balls, Phys. Rev. D, 98, 096016 (2018).

[37] M. N. Smolyakov, Perturbations against a Q-ball: Charge, energy, and additivity property, Phys. Rev. D, 97, 045011 (2018).

[38] M. I. Tsumagari, E. J. Copeland, and P. M. Saffin, Some stationary properties of a Q-ball in arbitrary space dimensions, Phys. Rev. D, 78, 065021 (2008).

[39] T.D. Lee and Y. Pang, Nontopological solitons, Phys. Rep, 221251 (1992).

[40] S. Coleman, Q-balls, Nucl. Phys. B, 262263 (1985).

[41] D. Bazeia, M. A. Marques, R. Menezes, Exact solutions, energy, and charge of stable Q-balls, Eur. Phys. J. C, 76, 241 (2016).

[42] D. Bazeia, L.Losano, et al, Split Q-balls, Physics Letters B, 765, 359 (2017).

[43] K. N. Anagnostopoulos, M. Axenides, et al, Large gauged Q balls, Phys. Rev. D, 64, 125006 (2001).

[44] M. Axenides, S. Komineas, et al, Dynamics of nontopological solitons: Q balls, Phys. Rev. D, 61, $085006(2000)$.

[45] P. Bowcock, D. Foster, and P. Sutcliffe, Q-balls, integrability and duality, J. Phys. A: Math. Theor, 42, 085403 (2009). 
[46] T. Shiromizu, T. Uesugi, and M. Aoki, Perturbation analysis of deformed Q-balls and primordial magnetic field, Phys. Rev. D, 59, 125010 (1999).

[47] T. Shiromizu, Generation of a magnetic field due to excited Q-balls, Phys. Rev. D, 58, 107301 (1998).

[48] D. Bazeia, L.Losano, et al, Compact Q-balls, Physics Letters B, 758, 146-151 (2016).

[49] N. Riazi, Wave-Particle Duality in Nonlinear Klein-Gordon Equation, Int. J. Theor. Phys, 50, 3451 (2011).

[50] M. Mohammadi, N. Riazi, Bi-dimensional soliton-like solutions of the nonlinear complex sineGordon system, Prog. Theor. Exp. Phys, 2014, 023 A03 (2014).

[51] M. Mohammadi, A. R. Olamaei, Soliton-like Solutions of the Complex Non-linear KleinGordon Systems in 1 + 1 Dimensions, IJAA, 4, 57-68 (2017).

[52] A. Saha and B. Talukdar, Rep. Math. Phys. 73, 299-309 (2014).

[53] Z. E. Musielak, Standard and non-standard Lagrangians for dissipative dynamical systems with variable coefficients, J. Phys. A: Math. Theor, 41, 055205 (2008).

[54] A.R. El-Nabulsi, Non-Linear Dynamics with Non-Standard Lagrangians, Qual. Theory Dyn. Syst, 12, 273 (2013).

[55] R. A. El-Nabulsi, Classical string field mechanics with non-standard Lagrangians, Math Sci, 9, 173-179 (2015).

[56] R. A. El-Nabulsi, Nonlinear integro-differential Einsteins field equations from nonstandard Lagrangians, Can. J. Phys. 92, 1149-1153 (2014). 